\title{
Particle tracking for polydisperse sedimenting droplets in phase separation
}

\author{
T. Lapp $\cdot$ M. Rohloff $\cdot$ J. Vollmer $\cdot$ B. Hof
}

Received: 22 June 2011/Revised: 9 November 2011/Accepted: 24 November 2011/Published online: 20 December 2011

(C) The Author(s) 2011. This article is published with open access at Springerlink.com

\begin{abstract}
When a binary fluid demixes under a slow temperature ramp, nucleation, coarsening and sedimentation of droplets lead to an oscillatory evolution of the phase-separating system. The advection of the sedimenting droplets is found to be chaotic. The flow is driven by density differences between two phases. Here, we show how image processing can be combined with particle tracking to resolve droplet size and velocity simultaneously. Droplets are used as tracer particles, and the sedimentation velocity is determined. Taking these effects into account, droplets with radii in the range of $4-40 \mu \mathrm{m}$ are detected and tracked. Based on these data, we resolve the oscillations in the droplet size distribution that are coupled to the convective flow.
\end{abstract}

\section{Introduction}

The characterization of particle distributions in fluids is important to control and optimize processes in food, pharmaceutical, oil and chemical industry (Heffels et al. (1998)). To determine the particle mass flux, particle sizes and velocities have to be measured simultaneously, which was achieved by Petrak (2002).

Here, we present a particle tracking algorithm, which uses droplets as marker particles to measure the flow field. They are created naturally in the phase separation process

T. Lapp ( $\varangle)$ · M. Rohloff · J. Vollmer · B. Hof

Max-Planck-Institute for Dynamics and Self-Organization,

37073 Goettingen, Germany

e-mail: juergen.vollmer@ds.mpg.de

T. Lapp · M. Rohloff · J. Vollmer

Faculty for Physics, University of Goettingen,

37077 Goettingen, Germany of demixing binary systems. Droplet positions and radii are detected simultaneously. The radius can therefore be used as a criterion to identify droplets in subsequent images. Assuming Stokes law, the sedimentation velocity can be calculated from the droplet radius. The droplet velocity is decomposed into sedimentation velocity and advection by the flow. By subtracting the sedimentation velocity from the droplet velocity, the advection of all droplets can be used to measure the flow field. With the advective flow field and the sedimentation velocity of each droplet, its position in the next frame can be predicted and compared to the image.

Previous studies have extensively used particle tracking velocimetry as a measurement technique to investigate turbulent flows carrying small particles (Maas et al. 1993; Malik et al. 1993; Ouellette et al. 2006; Kreizer et al. 2010). To that end, monodisperse tracer particles are added to the fluid whose position is detected by image processing of high-speed camera data. While this procedure is followed successfully for monodisperse particles in single-phase flows, it is not easily applicable for measuring flow patterns in demixing binary systems, which have been studied by Vollmer et al. (1997, 2002) and Emmanuel and Berkowitz (2006). Tracer particles cannot be added as they would act as nucleation centers and therefore affect the droplet number density. Furthermore, colloidal particles aggregate on the interfaces and change the growth and coalescence rate, as Thijssen and Clegg (2010) have shown. For a review of the stabilizing effect of colloidal particles in emulsions, see Binks (2002) and Aveyard et al. (2003).

There also is a broad range of acoustic/electroacoustic and optical techniques for the measurement of droplet size distributions, an overview given by Maass et al. (2009). Several laser-based techniques measure chord lengths that have to be transferred into droplet size distributions 
(Hu et al. 2006), for example, focus beam reflectance measurement (FBRM, see e.g. Ruf et al. 2000) or optical reflectance measurement (ORM, see e.g. Lovick et al. 2005). However, Maaß et al. (2011) and Simmons et al. (2000) have shown that these techniques give inaccurate results for liquid/liquid dispersions in comparison with image analysis of in situ microscope imaging.

To investigate the evolution of demixing systems, it is therefore preferential to use particle tracking of droplets present in the demixing system, as described in the following.

The article is organized as follows: We first characterize the chemical system. Then, we introduce the experimental setup with a focus on the illumination technique and explain the experimental procedure (Sect. 2). In Sect. 3, we present the particle tracking algorithm. In Sect. 4, we measure the sedimentation velocity of droplets and give an indication on the uncertainties in the radius detection. We then use the velocity information to filter out falsely detected droplets. We show how the droplet size distribution and the characteristics of the flow field evolve in time. Finally, in Sect. 5, we discuss the applicability and the limitations of the droplet tracking procedure for the investigation of demixing binary fluids.

\section{Experimental method}

\subsection{Model system}

As a model system, a mixture of water and isobutoxyethanol (i-BE) is chosen. It has a lower critical point at $25.5^{\circ} \mathrm{C}$ and demixes under heating. A phase diagram is shown in Fig. 1. The sample can easily be prepared at room temperature and demixes at temperatures between $25.5^{\circ}$ and $80^{\circ} \mathrm{C}$, as shown by Nakayama et al. (2001). The i-BE (purity $\geq 97 \%$ ) is purchased from Wako Chemicals $\mathrm{GmbH}$ and used without purification. The data points in the phase diagram (Fig. 1) were determined by turbidity measurements. The solid lines show fits with sixth-order (left branch) and fourth-order (right branch) polynomials, respectively.

The density and the viscosity of the mixture depend on composition as well as on temperature. For the density, the data of Doi et al. (2000) are used, taking thermal expansion and molar excess volume explicitly into account. The determination of the viscosity is described in the appendix.

Droplet detection is improved by using Nile Red as a fluorescent dye. Nile Red solved in butoxyethanol absorbs in the wavelength range of 500-590 nm (green light) and emits between 580 and $700 \mathrm{~nm}$ (red). The dye solves preferentially in organic compounds and poorly in water. Fowler and Greenspan (1985) point out that the fluorescence of Nile Red depends strongly on the polarity of the

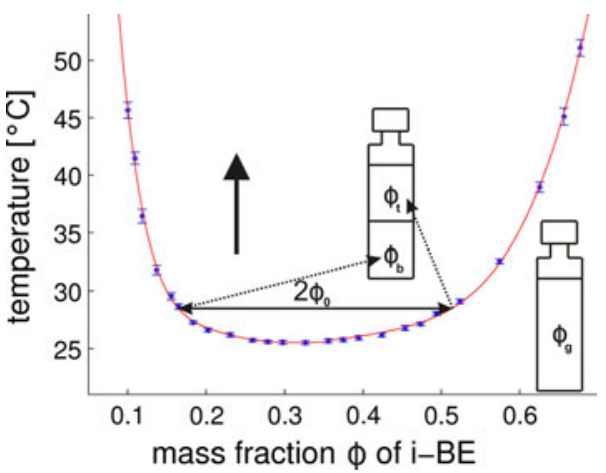

Fig. 1 Phase diagram of water/isobutoxyethanol mixture between $25^{\circ}$ and $50^{\circ} \mathrm{C}$. The samples are filled with the composition $\Phi_{\mathrm{g}}$. The mixture separates above a critical temperature $T_{\mathrm{c}}=25.5^{\circ} \mathrm{C}$. For slow temperature ramps, the compositions $\Phi_{\mathrm{b}}, \Phi_{\mathrm{t}}$ of the bottom and top phase follow the left and right branch of the binodal, respectively. $2 \Phi_{0}=\Phi_{\mathrm{t}}-\Phi_{\mathrm{b}}$ denotes the width of the miscibility gap. The left and the right branch are fitted with sixth- and fourth-order polynomials, respectively

solvent. In particular, its fluorescence is quenched in water. Therefore, the i-BE-rich phase appears bright and the water-rich phase stays relatively dark. Since each phase consists of a mixture of water and i-BE, the contrast between dark and bright parts in the images recorded during a measurement depends on the composition of the two phases. It was checked that the dye has no significant influence on the phase diagram.

\subsection{Experimental setup}

The probe is contained in a fluorescence cell $(10 \times 10 \times$ $33 \mathrm{~mm}$ ) 117.100F-QS made by Hellma GmbH. The measurement cell is mounted in a water bath with controlled temperature (see Fig. 2). An immersion cooler Haake EK20 is cooling with constant power, and a temperature control module Haake DC30 is heating the water bath to a preset temperature. Additionally, the temperature of the water near the sample is measured with a PT100 temperature sensor. The temperature is controlled with an accuracy of $15 \mathrm{mK}$.

To get enough signal from the fluorescently labeled i-BE-rich phase, a bright light source is required. A laser light sheet is not suitable for illumination since it is unidirectional and the two phases are not index matched. Each droplet acts as a little lens that focuses and diffracts the parallel laser light sheet. After passing a short distance of the sample, the droplets have transformed the uniform light sheet into a stripe pattern, which illuminates the droplets very inhomogeneously. In contrast, a mercury short arc lamp (LOT-Oriel, $100 \mathrm{~W}$ ) with a bright light emitting spot gives far better results. It provides a high light intensity, which is diffuse enough that almost no stripes appear (Fig. 3a). 


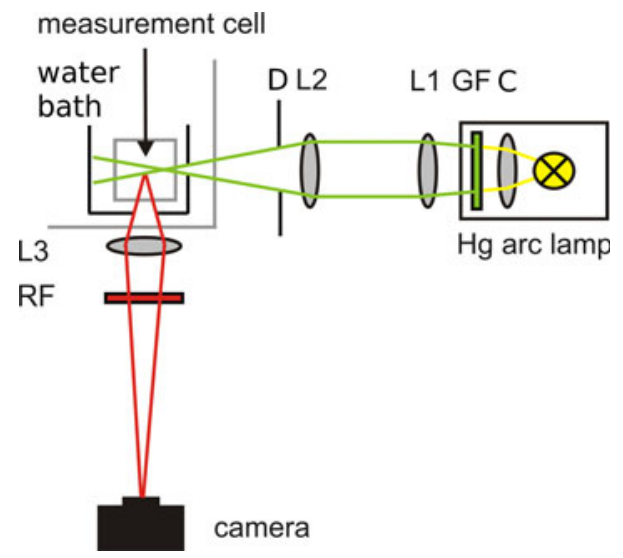

Fig. 2 Sketch of the experimental setup, top view. The light of a short arc $\mathrm{Hg}$ vapor lamp is collected by a collimator lens (C) and parallelized by a spherical lens (L1). A cylindrical lens (L2) forms a light sheet that is cut by a diaphragm (D) and a line aperture $(300 \mu \mathrm{m}$ width) directly in front of the sample cell. The light sheet illuminates a plane of the binary mixture, and a green filter (GF) selects the wavelength range suitable for excitation of the dye. The emission of the fluorescently labeled phase passes a red filter (RF) and is projected by an objective (L3) onto a $5 \mathrm{Mp} \mathrm{CCD}$ camera chip. The sample cell is mounted in a water bath with controlled temperature

A sketch of the optical components is given in Fig. 2. The light is collected by the collimator lens (C), and a bandpass filter (GF) selects the green emission lines (546 and $577 / 579 \mathrm{~nm}$ ) for the excitation of the fluorescent dye. The spherical lens $(\mathrm{L} 1, f=200 \mathrm{~mm})$ forms a parallel light beam. The cylindrical lens (L2, $f=80 \mathrm{~mm}$ ) focuses the light normal to the focal plane of the camera. A spherical diaphragm (D) allows to adjust the amount of light manually. With a narrow slit of $300 \mu \mathrm{m}$ width directly in front of the measurement cell, a vertical light sheet is formed. The measurement cell is covered by black apertures to shield stray light. The fluorescent light from the illuminated plane of the sample is projected by a $f=35 \mathrm{~mm}$ objective (L3) to the chip of a BM-500CL monochrome progressive scan CCD camera. It takes $2,058 \times 2,448$ pixel images with a maximum frame rate of $15 \mathrm{~Hz}$, covering $1.3 \mathrm{~mm} \times 1.5 \mathrm{~mm}$ of the sample $(1.6 \mathrm{pixel} / \mu \mathrm{m})$. With a longpass filter (RF, edge $594 \mathrm{~nm}$ ), the excitation light is filtered.

The camera, temperature control and magnetic stirrer are connected to a computer. Measurements are fully automated using a LABVIEW program.

\subsection{Experimental procedure}

The temperature protocol for our experimental procedure is adapted from Auernhammer et al. (2005). After mixing the sample at $24^{\circ} \mathrm{C}$ for one hour, the temperature is set to $T=25.8^{\circ} \mathrm{C}(0.3 \mathrm{~K}$ above critical point $)$, and the system equilibrates for four hours. Then, a temperature ramp is run from $25.8^{\circ}$ to $50^{\circ} \mathrm{C}$ within $0.15-32 \mathrm{~h}$, which is designed to
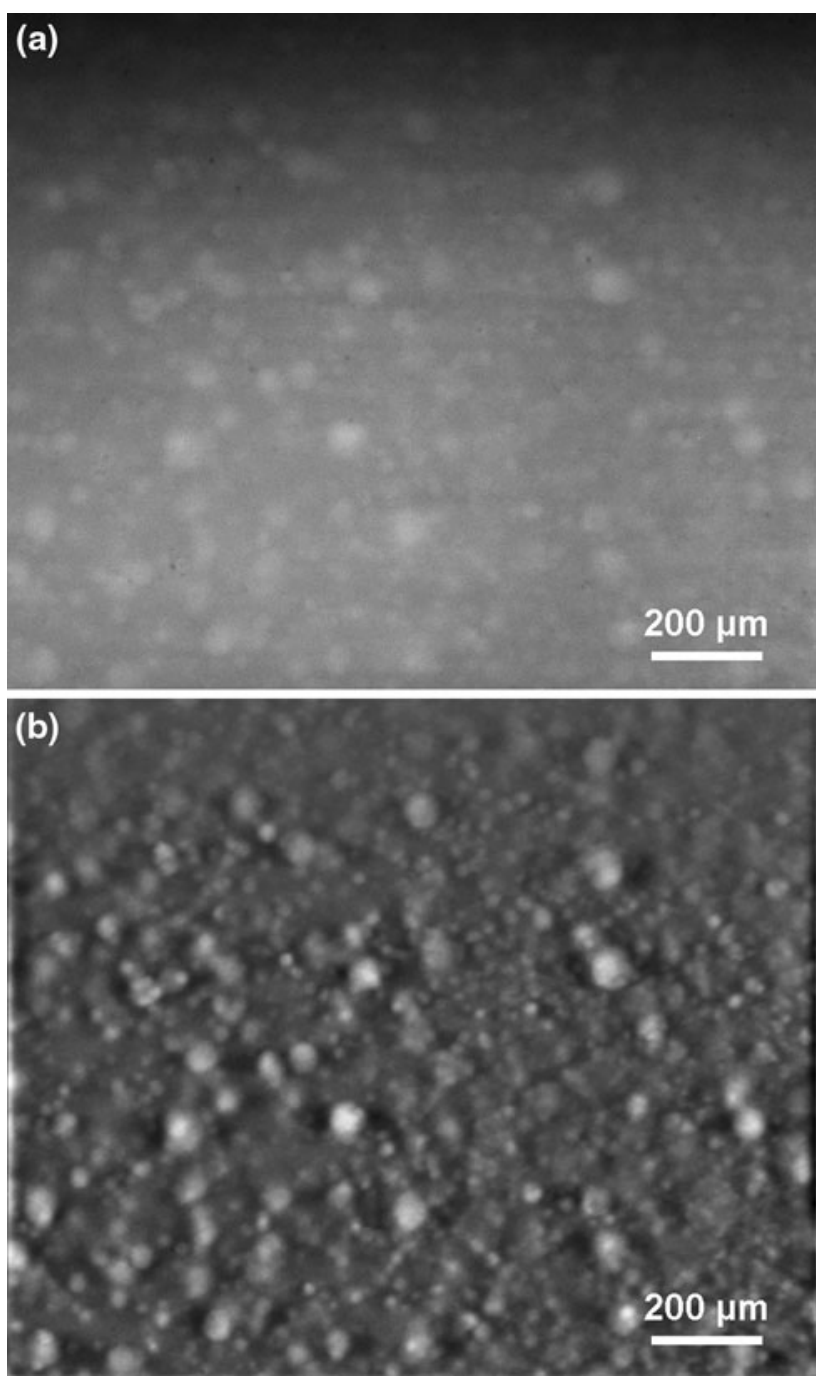

Fig. 3 a Raw image and $\mathbf{b}$ preprocessed image of i-BE-rich droplets in the water-rich bottom phase with $\xi=1.05 \times 10^{-5} \mathrm{~s}$ at $25.98^{\circ} \mathrm{C}$ $\pm 0.02^{\circ} \mathrm{C}$. Horizontal stripes and the vertical intensity gradient are removed by Fourier filtering

keep the volume flux between the phases constant. The temperature of the thermostat is set in steps of $0.01 \mathrm{~K}$ with a rate of $1 \mathrm{~Hz}$ according to the temperature ramp calculated below.

Following Auernhammer et al. (2005) and Cates et al. (2003), a driving parameter $\xi$ is defined

$\xi=\frac{1}{\Phi_{0}(T(t))} \frac{\partial \Phi_{0}(T(t))}{\partial t}$

with $2 \Phi_{0}=\Phi_{\mathrm{t}}-\Phi_{\mathrm{b}}$, where $\Phi_{\mathrm{t}}$ and $\Phi_{\mathrm{b}}$ denote the compositions of the top and the bottom phase, respectively (compare Fig. 1). Hence, $2 \Phi_{0}(T)$ is the width of the miscibility gap for a given temperature $T$. The driving parameter $\xi$ is equal to the rate of droplet mass production in the two phases. Equation 1 is inverted to calculate a temperature ramp with fixed driving, 
$T(t+\delta t)=T(t)+\frac{\xi \Phi_{0}(T(t))}{\partial_{T} \Phi_{0}(T(t))} \delta t$.

Equation 2 is integrated with 10,000 uniformly distributed time steps in the range $\left[t_{0}, t_{\text {end }}\right]$ chosen such that $T\left(t_{0}\right)=25.8^{\circ} \mathrm{C}$ and $T\left(t_{\text {end }}\right)=50.0^{\circ} \mathrm{C}$.

\section{Particle detection and tracking}

In this section, we describe a particle detection and tracking algorithm for polydisperse sedimenting particles. Radii and positions are detected simultaneously. The first part describes an image processing algorithm, which detects droplets in separate images. In the next step, the flow field is estimated. Then, the droplets are tracked through a series of images. Finally, the velocity field is recalculated based on the droplet trajectories. This procedure allows us to sort out artifacts (caused, e.g., by dirt or overlaps of droplet images) of the image processing and to determine Lagrangian particle velocities. The image processing is done with the MATLAB Image Processing Toolbox. The data (typically 20,000 images per measurement) are processed on a computer cluster. For simplicity, we restrict our description to the detection of the fluorescently labeled droplets in the bottom phase. By inverting the images and the direction of gravity, the same algorithm can be used for the dark droplets detected in the top phase.

\subsection{Processing of single images}

\subsubsection{Preprocessing}

In a first step, dark spots caused by dirt on the camera chip are removed with a flat field correction. Next, the image is Fourier filtered and the contrast is optimized. To reduce the horizontal stripes (illumination from the right) produced by the light dispersion of the droplets, a high-pass filter (Gaussian filter with width 5 in horizontal and 200 in vertical direction) is applied. For reducing the noise, a lowpass filter (isotropic Gaussian filter, $\sigma=100$ ) is applied. In Fig. 3, a raw image and a preprocessed image of fluorescent i-BE-rich droplets in the denser water-rich bottom phase are shown. For high droplet densities, there is a significant number of overlaps of droplet images. Therefore, we need special algorithms to take care of this for detection.

\subsubsection{Droplet detection}

The droplets are detected in the preprocessed image using two strategies. The first relies on thresholding the image, and the other uses marker-controlled watershed segmentation. The first strategy is visualized in Fig. 4 giving an example. All pixels darker than a threshold level (Fig. 4a) are identified as background (Fig. 4b). Isolated background regions are deleted. Then, for each pixel, the distance to the background is calculated (Fig. 4c). Local maxima in this distance map correspond to droplet centers, their distance to the background being their radius (Fig. 4d). This procedure is carried out twice with two thresholds, one being better for finding big droplets the other for small droplets.

The second strategy is based on the marker-controlled watershed segmentation algorithm proposed by Gonzalez et al. (2004, p. 422). It is illustrated in Fig. 5. Extended maxima in the image (Fig. 5a) are used as marker for the droplets (Fig. 5b). Lines between these maxima as well as extended minima are markers for the background. With a Sobel filter, the intensity gradient in the image is calculated (Fig. 5c). The markers are imposed as minima on the gradient image upon which a watershed transform is operated. The watershed lines of the intensity gradient correspond to droplet edges. The markers are needed to avoid over-segmentation. Areas and centroids of the regions enclosed by the watershed lines are taken for the droplet radii and positions (Fig. 5d).

The two different approaches have different performance depending on the droplet arrangement in the images: The threshold-based approach works very well for big droplets, independent of overlaps with other droplets. The result especially for the position but also for the radius is usually quite good. However, small droplets on top of bigger ones cannot be detected this way. This is the reason to use also the watershed segmentation-based algorithm. On the one hand, it usually has difficulties in detecting the big droplets properly, as they are typically quite inhomogeneous in intensity. This often leads to a segmentation of big droplets into smaller ones. On the other hand, small droplets are found quite reliably, even when they overlap with bigger droplets (as illustrated in the sketch of Fig. 5d). Position and radius are finally determined by the match to the image, which will be described in Sect. 3.1.3. Hence the result does not depend too much on the algorithm the droplet was detected with.

Using these two approaches, even small droplets on top of bigger ones and overlapping images of droplets can be resolved. However, some droplets are found multiple times, and some imaging artifacts are taken for droplets. If two droplets with radii $r_{1}, r_{2}$ are detected close to each other (centroid distance less than one radius) with almost the same radius (ratio $0.7<r_{1}$ / $r_{2}<1.4$ ), they are considered as two detections of the same physical droplet. 
Fig. 4 Sketch of distance-based image segmentation. a False color plot of two overlapping images of droplets.

b Thresholded image.

c Distance from background. d Detected droplets (black) based on local maxima of distance
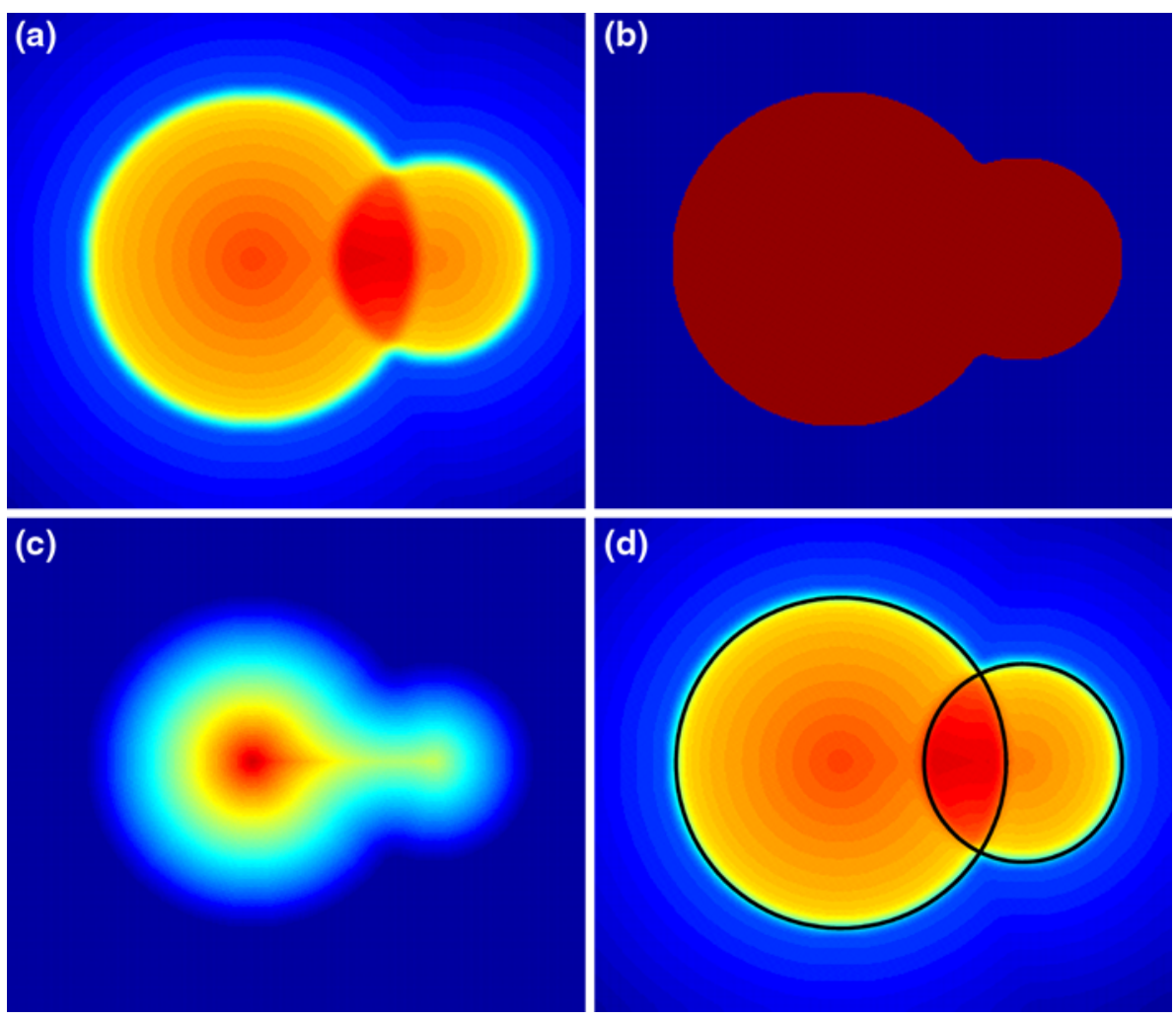

Fig. 5 Sketch of watershed image segmentation. a False color plot of two overlapping images of droplets. b Bright extended maxima mark droplets, lines in between and dark regions mark background. c Markers are imposed as minima (blue) on gradient image. Watershed lines follow droplet edges (red). d Detected droplets (black) based on regions enclosed by watershed lines
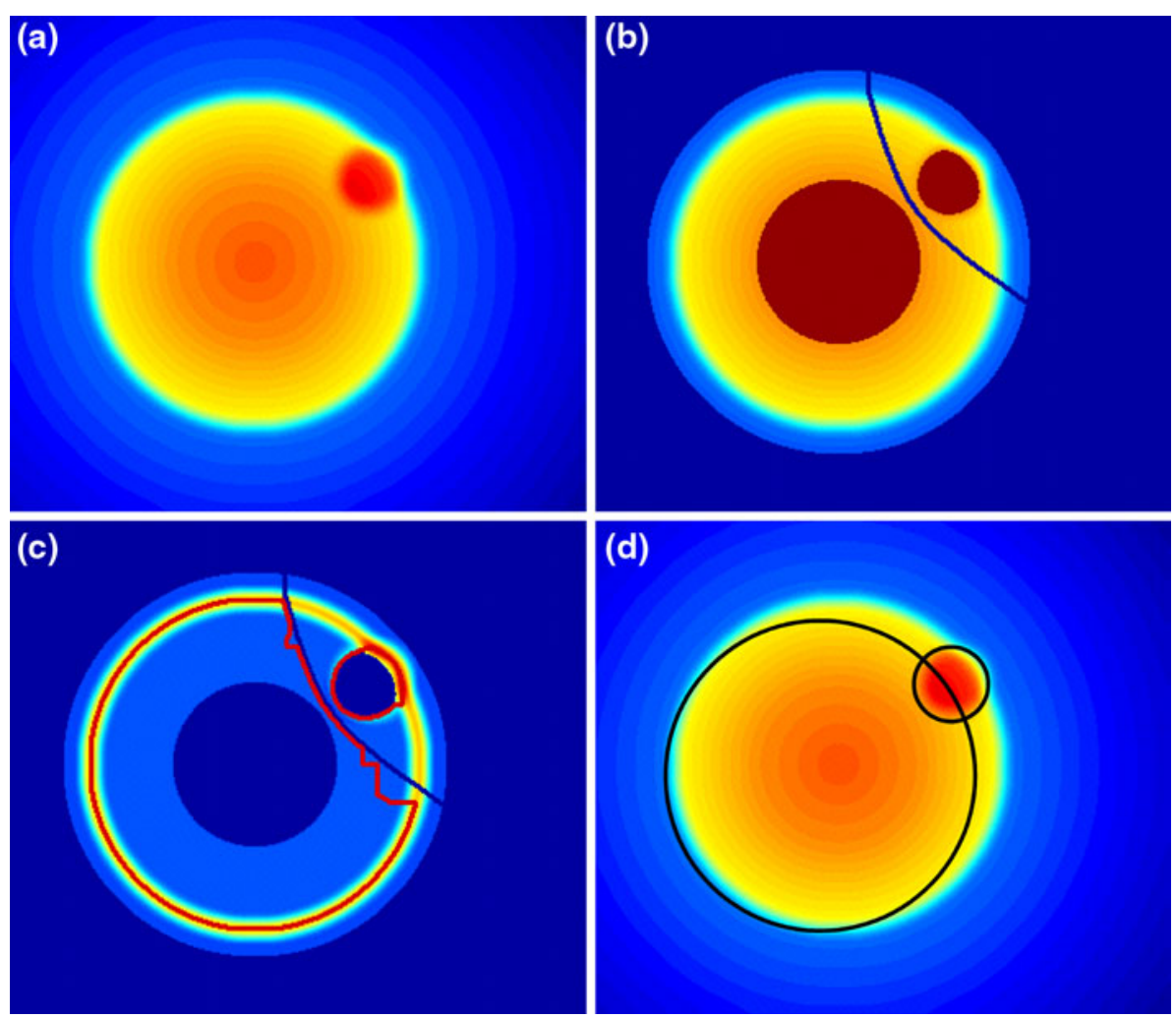


\subsubsection{Matching to image}

Not fully compensated dirt on the camera chip, overlaps of droplet images or unsharp droplet images can cause false detections. Hence, a second step is implemented to distinguish droplets from artifacts, and the estimates of the droplet radius $r$ and position $(x, y)$ [pixels] are compared to the preprocessed image. As characteristics of droplets in the image, we consider them being brighter than their environment and having a circular intensity gradient at the edge (Fig. 6). A match function $m$ is defined as

$$
\begin{aligned}
m(x, y, r)= & A(r)\left[I_{\text {drop }}(x, y, r)-I_{\text {ring }}(x, y, r)\right] \\
& -B \frac{\left(x-x_{\mathrm{r}}\right)^{2}+\left(y-y_{\mathrm{r}}\right)^{2}}{r^{2}}
\end{aligned}
$$

where the radius $r$ and the coordinates $x, x_{r}, y$ and $y_{r}$ are given in pixels. The difference between the mean intensity of the droplet $I_{\mathrm{drop}}(x, y, r)$ and the mean intensity $I_{\text {ring }}$ $(x, y, r)$ of a ring of 3 pixels width around it measures the intensity gradient at the droplet edge (Fig. 6a). An empirical radius-dependent prefactor $A(r)=1 /\left(30+r^{1.3}\right)$ ensures comparable matches for small and big droplets. Often, small droplets are detected erroneously at the edge of big droplets. In this case, a little part of its halo is covering the dark background, and the remaining part is covering the big droplet. The intensity-weighted centroid of the halo (denoted as $x_{\mathrm{r}}$ and $y_{\mathrm{r}}$ ) is calculated and compared to the droplet position (Fig. 6b). The deviation accounts for this asymmetry and reduces the value of the match. It is weighted with the empirical prefactor $B=3$. For every droplet found in the droplet detection step, the match with the image is calculated. By varying radius and position of the droplet, a local maximum of the match is found. All droplets with a match bigger than 0.08 are used for further analysis.

A final step to increase the reliability of the measured size distribution takes advantage of the time information of image sequences. Tracking the droplets allows to sort out

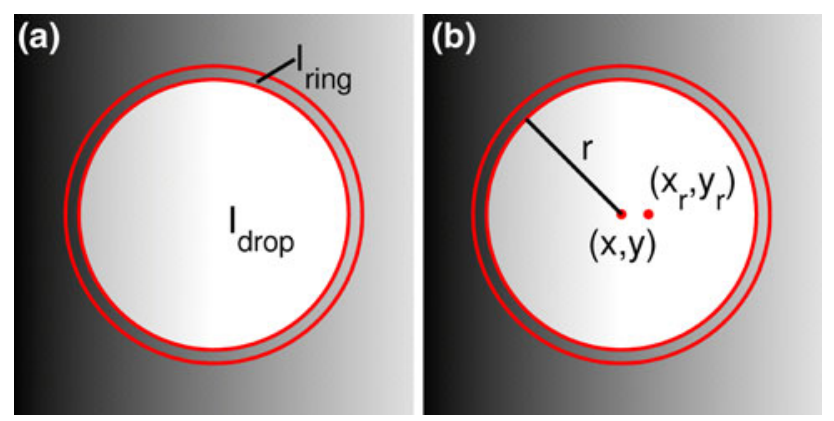

Fig. 6 Definition sketch for the match function. a $I_{\text {drop }}$ denotes the mean intensity of the droplet, $I_{\text {ring }}$ the intensity of a ring around it. b $(x, y)$ is the centroid of the droplet with radius $r,\left(x_{\mathrm{r}}, y_{\mathrm{r}}\right)$ is the intensity-weighted centroid of the ring unphysical trajectories of artifacts and to find undetected droplets in the image using its past or future trajectory. To implement this information in Sect. 3.3.3, we must evaluate the flow field and the droplet trajectories in the flow.

\subsection{Calculation of flow field}

\subsubsection{Identification of droplets in consecutive images}

Typically, droplets with radii $4 \mu \mathrm{m}<r<40 \mu \mathrm{m}$ are detected. While small droplets $(r<10 \mu \mathrm{m})$ closely follow the flow, large droplets $(r>20 \mu \mathrm{m})$ are mainly driven by their buoyancy and do not qualify as tracers. They sediment toward the interface due to the density difference of the two phases. In a Lagrangian frame, co-moving with the surrounding fluid, the sedimentation velocity $u_{\text {sed }}$ amounts to the Stokes velocity for a sphere of radius $r$, which is (Batchelor 2005, p. 234)

$u_{\text {sed }}=\frac{2}{9} \frac{\Delta \rho g r^{2}}{\eta}$.

Here $\Delta \rho$ denotes the mass density difference between sphere and fluid; $g$, the gravitational acceleration; and $\eta$, the dynamic viscosity of the fluid. Hence, the droplet velocity $u_{\text {drop }}$ can be split into a sedimentation and an advection term

$u_{\text {drop }}(x, y, r, t)=u_{\text {sed }}(r)+u_{\text {flow }}(x, y, t)$.

This approach will be checked in Sect. 4.1. To track droplets, the corresponding images of droplets in consecutive frames must be identified. To this end, the velocity field of the previous time step $u_{\text {flow }}(x, y, t-\delta t)$ is taken as an initial guess for $u_{\text {flow }}(x, y, t)$. For each droplet found in one image, the position in the next image is predicted using Eq. 5. The prediction is compared to the droplets found in the image, and the droplet pair with the closest distance between prediction and actual position and with similar radii is identified as one physical droplet. We find that the growth of droplets (about $1.5 \mu \mathrm{m}$ between successive images for big droplets) is smaller than the error in the radius detection (3.8 $\mu \mathrm{m}$ for big droplets, cf. Sect. 4.4). Therefore, it does not affect the tracking result. In Fig. 7, the droplets in the image are marked with red circles, and the corresponding droplets in the next image, with green circles. The procedure used to determine the flow field, which is indicated by the arrows, is lined out in Sect. 3.2.2. Subsequently in Sect. 3.3, we describe details of the scheme adopted for finding particle trajectories.

\subsubsection{Determine the flow field}

In order to calculate the Eulerian flow field $u_{\text {flow }}(x, y, t)$, the image is sampled by an equidistant mesh with $25 \times 20$ 


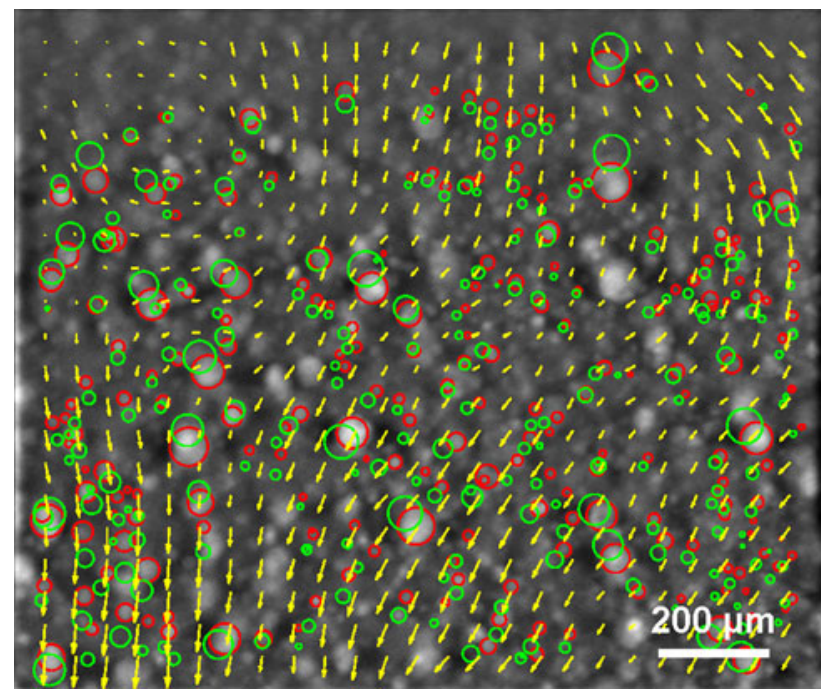

Fig. 7 Fluorescently labeled droplets in the bottom phase (Fig. 3b). The arrows correspond to the flow field. Red circles mark the droplets found in the image, and green circles, their position and radius in the next image

grid points. The grid distance is about $60 \mu \mathrm{m}$, which is in the order of the droplet distance. The correlation length of the flow field is about $200 \mu \mathrm{m}$, so the grid can resolve the flow structures. A flow correlation time of $30 \mathrm{~s}$ allows for small frame rates (order of $1 \mathrm{~Hz}$ ). By subtracting the sedimentation velocity $u_{\text {sed }}$ from the droplet velocity $u_{\text {drop }}$, the flow contribution $u_{\text {flow }}$ can be calculated: The advection of the $N$ droplets in each mesh cell $(i)$ is averaged, incorporating with half weight the $M$ droplets of neighboring cells $(i j)$ :

$$
\begin{aligned}
u_{\text {flow }}^{(i)}= & \frac{1}{N+M / 2} \\
& \times\left[\sum_{k=1}^{N}\left({ }^{k} u_{\text {drop }}^{(i)}-{ }^{k} u_{\text {sed }}^{(i)}\right)+\frac{1}{2} \sum_{k=1}^{M} \sum_{j=1}^{4}\left({ }^{k} u_{\text {drop }}^{(i j)}-{ }^{k} u_{\text {sed }}^{(i j)}\right)\right]
\end{aligned}
$$

\subsubsection{Smoothing of flow field}

The advection field $u_{\text {flow }}^{(i)}$ is smoothed with a weighted average of next-nearest neighboring cells to eliminate unphysical discontinuities.

$$
\bar{u}_{\text {flow }}^{(i)}=\frac{1}{4} u_{\text {flow }}^{(i)}+\underbrace{\frac{1}{8} \sum_{j=1}^{4} u_{\text {flow }}^{(i j)}}_{\text {next neighbors }}+\underbrace{\frac{1}{16} \sum_{j^{\prime}=1}^{4} u_{\text {flow }}^{\left(i j^{\prime}\right)}}_{\text {next-nearest neighbors }}
$$

In Fig. 7 a smoothed advection field is shown together with the matched droplets. The Eulerian flow field is used to predict the droplet positions in the next image. Note that the spatial distribution of detected droplets is not homogeneous and some grid points can only be calculated by interpolation. For the subsequent analysis of the flow field, only the grid points that are directly computed from droplet displacements are taken into account.

\subsection{Particle tracking}

\subsubsection{Forward tracking}

Now that the advection field and sedimentation velocities for each droplet found in the image at time $t$ have been determined, the position at time $t+\delta t$ can be predicted. This prediction is matched to the image of $t+\delta t$ and a local maximum of the match is searched for. This procedure is illustrated in Fig. 8. Having determined the displacement from time $t$ to $t+\delta t$, the advection field can be recalculated. In the next step, the positions of droplets tracked from image $t$ to $t+\delta t$ plus all other droplets also detected in image $t+\delta t$ are predicted for the time step $t+2 \delta t$. They are compared to the droplets in image $t+$ $2 \delta t$, and the procedure is repeated. The identified droplet pairs are sorted into trajectories, which contain the position and radius values for the different frames. Based on the trajectories, the flow field $u_{\text {flow }}(x, y, t)$ is recalculated according to the procedure described in Sect. 3.2.2.

\subsubsection{Backward tracking}

The forward tracking result can be improved by subsequently tracking the droplets backward in time. The positions of droplets at the beginning of the trajectories are predicted for the time step before and matched to the image (compare Fig. 9). The droplet is compared to the entries in the other trajectories to merge interrupted trajectories and to suppress branching of trajectories. As yet, our tracking algorithm does not account for the collision of droplets.

\subsubsection{Filtering of trajectories}

Trajectories shorter than three time steps are removed. Finally, all droplets that have moved by less than half the average advection velocity during the tracked time interval are deleted. Most likely, those spots are imaging artifacts. Eventually, the flow field is recalculated once more based on the enlarged and filtered trajectories in order to arrive at a consistent data set.

\subsubsection{Depth of measurement volume}

The threshold of the circular intensity gradient (cf. Sect. 3.1.3) has to be chosen such that very unsharp droplets are discriminated. Eventually, both the threshold for the intensity gradient and the "trackability criterion" for droplets determine the effective measurement volume. We find that the depth, in which a droplet can be detected, is 
Fig. 8 Sketch of the forward tracking algorithm.

a Sedimentation velocity (blue, upward) and advection (green, 2 o'clock), b predict the next position (red dashed), $\mathbf{c}$ match the prediction to the next image (red solid) and $\mathbf{d}$ build trajectory (black)

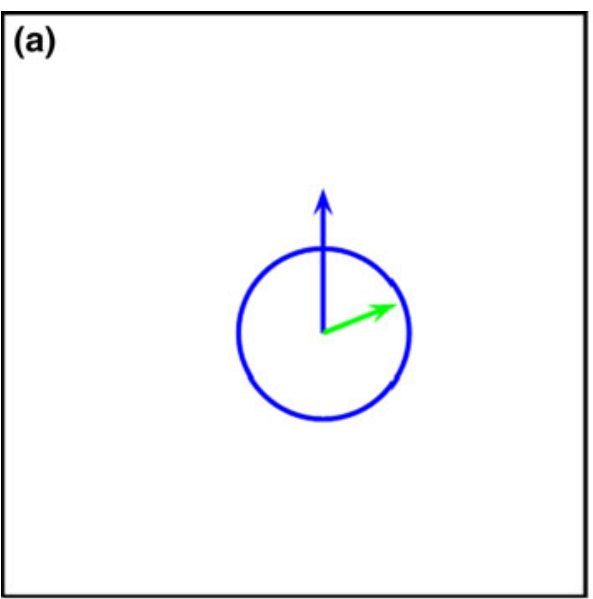

(b)

(c)

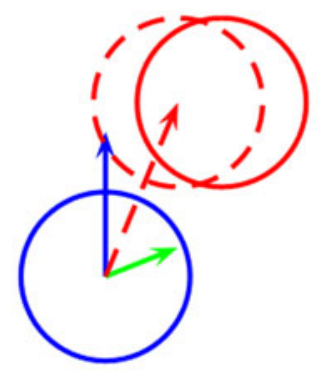

(d)
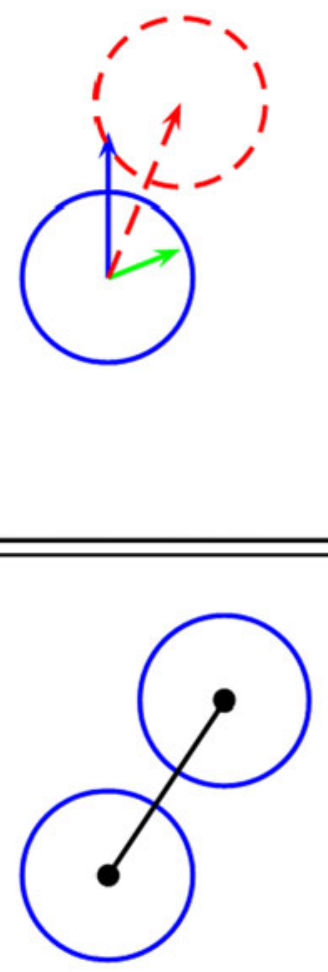

proportional to its diameter. The droplet-size-dependent measurement volume has to be accounted for in the evaluation of the size distribution. For the computation of the droplet number density, the effective measurement volume (image width $\times$ image height $\times 2$ droplet diameters) has been used. This serves as a radius-dependent bias correction function to get the droplet number density from the number of detected (and tracked) droplets.

The plausibility of the measured droplet volume densities can be tested by a physical consideration. The driving rate $\xi$ determines the rate at which droplet volume is produced during the temperature ramp. Comparing the measured droplet volume fraction to the volume production due to the temperature change gives a fair agreement. We are therefore quite confident in the measured droplet size distributions.

\section{Results}

\subsection{Sedimentation velocities}

We first aim to confirm the validity of the approach Eqs. 4, 5. In Fig. 10, the vertical velocity calculated from the trajectories of the tracked droplets is shown as a function of the radius (red data points). The statistical error of the mean vertical droplet velocities is smaller than the marker size in the figure. The droplet velocities are compared to the sedimentation velocity (blue points) due to Eq. 4 modified by the mean vertical velocity of the flow, which is calculated from the flow field. The error bar of the Stokes Eq. velocity computation represents the deviation of density difference and viscosity in the considered time interval due to the temperature shift. The Stokes velocity of a sphere Eq. 4 appears to describe the average settling rate very well. We attribute the deviations for small droplet radii to the fact that both the droplet size distribution and the average flow velocity oscillate. Most of the small droplets can be found at the beginning of the oscillations, when the average downward flow velocity is relatively small (see below).

\subsection{Stokes number}

Equation 5 holds for droplets with a Stokes number St $\ll 1$. The Stokes number is defined as the ratio of two timescales

$\mathrm{St}=\frac{\tau_{\text {drop }}}{\tau_{\text {flow }}}$. 
Fig. 9 Sketch of the backward tracking algorithm:

a Sedimentation velocity (blue, upward) and advection (green, 8 o'clock), b predict the previous position (red dashed), c match the prediction to the previous image (red solid) and $\mathbf{d}$ enlarge the trajectory $($ black)
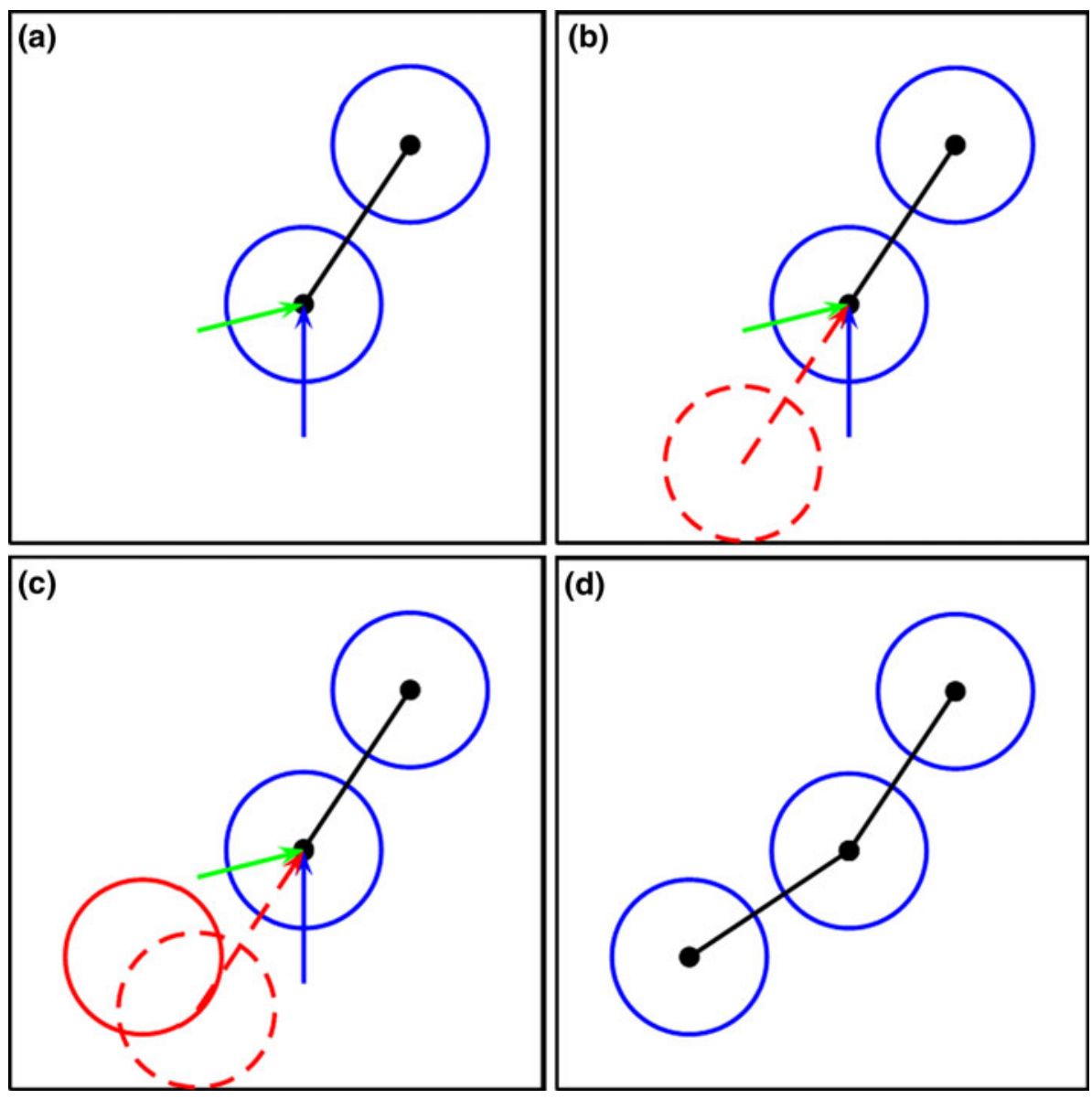

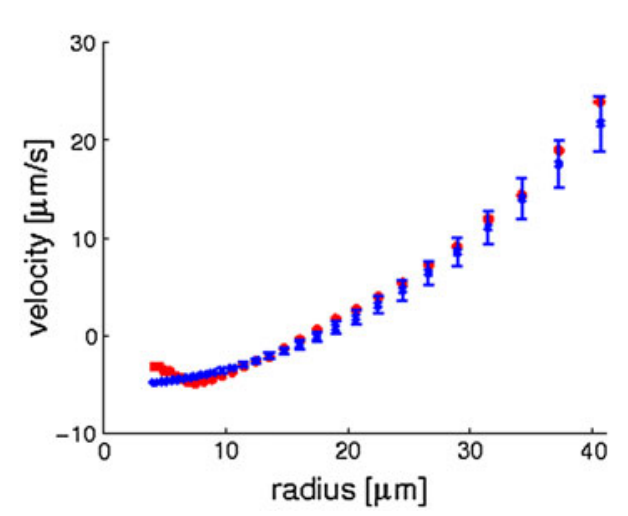

Fig. 10 Comparison of the measured vertical velocity of the droplets (red) and the Stokes velocity modified by the mean flow (blue), in the bottom phase at $26^{\circ} \mathrm{C}$. The error bar of the Stokes velocity corresponds to the deviation of density and viscosity within the investigated time interval. The statistical error of the mean vertical velocity (red) is smaller than the marker size. The systematic deviation for small droplets may be attributed to the fact that most small droplets are observed at times of small mean vertical flow velocity

In a flow which changes on the timescale $\tau_{\text {flow }}$ the advected droplets respond to accelerations on the timescale $\tau_{\text {drop }} \equiv \frac{2 \Delta \rho r^{2}}{9 \eta}$.

The correlation time of the flow is found to be $\tau_{\text {flow }} \sim 30 \mathrm{~s}$. As an upper limit for the droplet response time, we find $\tau_{\text {drop }}=36 \mu$ s at $T=50^{\circ} \mathrm{C}$ for droplets with $a=40 \mu \mathrm{m}$. Therefore, typical Stokes numbers in the experiment are smaller than $10^{-6}$. Even for a flow with high turbulence intensity with a Kolmogorov timescale of the order of milliseconds, the Stokes number would not exceed $10^{-3}$. Due to the small density differences of fluids, kinematic particle-turbulence interactions (for a review see Vaillancourt and Yau 2000) can hence be ruled out for droplets, even in a turbulent flow.

\subsection{Trajectory length}

For a quantitative analysis, trajectories of 2,000 images are sorted into radius bins according to their average radius. In Fig. 11, for each bin, the number of trajectories of a given length is color coded on a decade logarithmic scale. The red line indicates the time $t=H / u_{\text {sed }}$ needed by a droplet settling with Stokes velocity to pass through the image of height $H$. It puts an upper bound on the trajectory length 


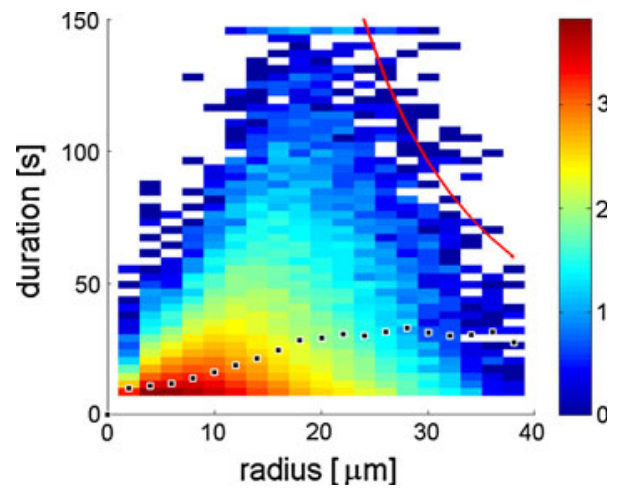

Fig. 11 Radius dependence of the trajectory length: The number of trajectories with a given radius and length is color coded on a logarithmic scale. The black squares denote the average length for each radius bin, and the solid red line is the sedimentation boundary. The duration of droplet observation increases roughly linear in time for droplets $\lesssim 20 \mu \mathrm{m}$. For bigger droplets, the observation is limited by the time droplets need to sediment through the imaged volume

and leads to a decrease in the trajectory length for big radii. The average length of droplet trajectories is plotted with black squares.

For small radii, the duration of individual droplet detection is observed to increase roughly linearly with the radius. Hence, we assume that the depth, in which a droplet can be detected, is proportional to its radius. Small droplets have to be exactly in the focal plane of the camera to be detectable, whereas big droplets can still be detected when their center is slightly off the focal plane. Since the radius is detected by the maximum of the intensity gradient, it is rather robust to slight blurring, and once the droplets are too far from the focal plane, they are no longer detected because the intensity gradient at their edge is below the threshold of the match function.

\subsection{Uncertainty in radius detection}

The fluctuations of the detected droplet radii in the trajectories are calculated to estimate the uncertainty of the attributed radius, which is presented in Fig. 12. For radii $r \leq 19 \mu \mathrm{m}$, a linear increase in the standard deviation is observed, yielding a constant relative uncertainty of about $20 \%$. Above $19 \mu \mathrm{m}$, the standard deviation is more or less constant with $3.8 \mu \mathrm{m}$. We take this as a measure for the uncertainty in radius detection.

It is worth to note that the ratio of the focal depth to the object distance is smaller than $1 \%$. Therefore, the magnification is constant within $1 \%$. Hence, the error in the detection has to be attributed to limitations of the image processing algorithm. Out-of-focus droplets that are visible in the images are dealt with in the following ways: The radius of the highest intensity gradient is used to determine the droplet radius. The position of the maximum in the

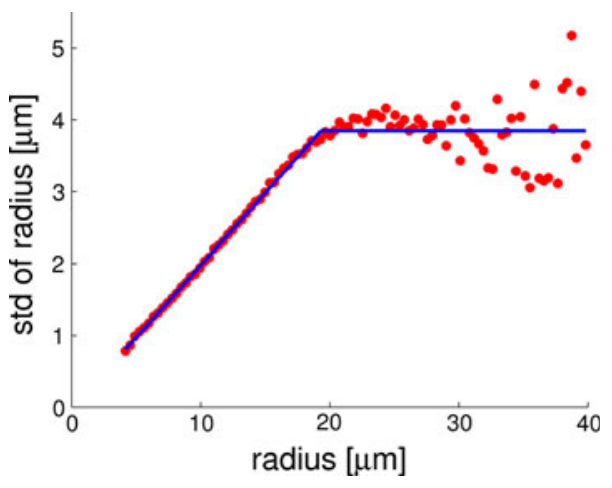

Fig. 12 For each tracked droplet, the standard deviation of the detected droplet radius is calculated. This serves as an estimate of the uncertainty in radius detection. For small droplets, the uncertainty is about $20 \%$ of the droplet radius, and at $20 \mu \mathrm{m}$ it levels of to $3.8 \mu \mathrm{m}$ (blue line)

intensity gradient does not change significantly when the droplet is only slightly unsharp. Droplets that are too unsharp are discarded by the criterion that the intensity gradient has to exceed a certain threshold. Secondly, only droplets that can be tracked through a sequence of images contribute to the measurement of the size distribution which discriminates almost all artefacts due to unsharp objects in the image.

\subsection{Oscillating size distribution of droplets}

By slowly heating the system, more than twenty oscillations in the size distribution can be found (Fig. 13). A complex interplay of nucleation (Krishnamurthy and Goldburg 1980; Sagui and Grant 1999; Vollmer 2008), coarsening (Aarts et al. 2005; Vollmer et al. 2007) and sedimentation (Hayase et al. 2008) causes oscillations of the supersaturation and turbidity, modeled by Vollmer et al. (2007) and Benczik and Vollmer (2010). As expected based on turbidity oscillations observed by Vollmer et al. (2002) and Auernhammer et al. (2005) in a multitude of binary mixtures subjected to the same temperature driving, they can also clearly be identified in plots of the droplet size distribution displayed in Fig. 13. Comparing the two size distribution plots in Fig. 13 reveals the effectiveness of droplet tracking. The noise in the processing of single images is reduced significantly, and the evolution of the size distribution is unraveled for small droplets also.

A detailed analysis of the oscillating size distribution and its analogy to precipitation cycles of other systems due to thermodynamic driving will be given elsewhere. Here, we only briefly comment on its origin: At the beginning of an oscillation, only very few droplets are present, and the supersaturation increases due to the temperature change. A nucleation wave sets in with subsequent diffusional growth of the droplets. When the droplets are grown to about 

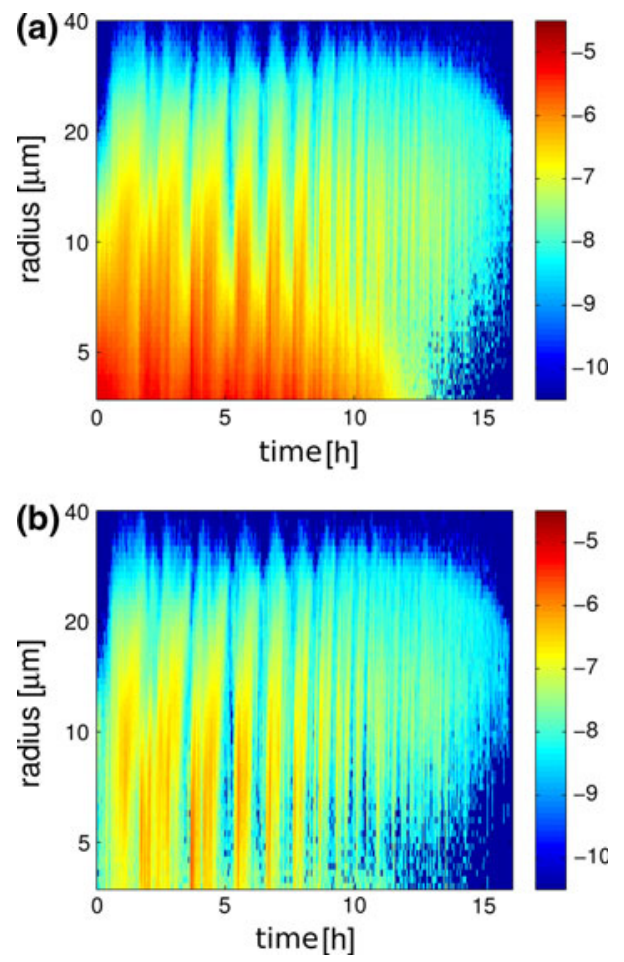

Fig. 13 Size distribution of droplets of the bottom phase with $\xi=$ $1.05 \cdot 10^{-5} \mathrm{~s}^{-1}$ : a before and $\mathbf{b}$ after consistency check based on droplet tracking. The droplet number density per radius given in $\mu \mathrm{m}^{-4}$ is color coded on a decadic logarithmic scale. The droplet distribution undergoes repeated precipitation cycles of nucleation, diffusional growth, coalescences and sedimentation

$10 \mu \mathrm{m}$ radius, they start to sediment and collide with smaller droplets in their path. Sedimentation and collisions reduce the droplet number density. Eventually, after almost all droplets have sedimented, a new nucleation wave sets in. Since nucleation of droplets is restricted to a narrow time window within the period, oscillation amplitudes of several orders of magnitude are observed.

\subsection{Oscillating flow properties}

To characterize the time evolution of the flow field (using only non-interpolated grid points), the mean vertical and horizontal flow velocities as well as the root mean squared velocity components are calculated and displayed in Fig. 14. For averaging in time, four bins for each oscillation are used. The mean horizontal velocity fluctuates around zero as expected for symmetry reasons, and the vertical flow velocity is typically negative. This can be explained by two effects. On the one hand, the upward sedimenting droplet volume has to be balanced by a downward fluid motion to ensure a zero net volume flux. On the other hand, there is a large scale convection since the system is heated from outside. Close to the walls, the compositions of the phases are slightly more separated. Hence, the density of the bulk is higher close to the

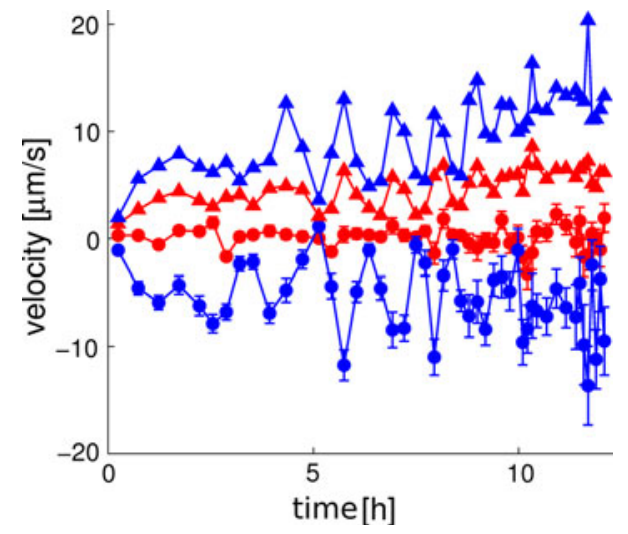

Fig. 14 Mean fluid velocity in horizontal (red circles) and vertical (blue circles) direction, and the rms velocity in horizontal (red triangles) and vertical (blue triangles) direction. Data of twelve oscillations are shown, where four consecutive data points correspond to one oscillation. The measurement is performed in the bottom phase with $\xi=1.05 \cdot 10^{-5} \mathrm{~s}^{-1}$

wall than in the central region of the sample. This drives a convection pattern with downward motion close to the wall. A coupling of the oscillating droplet size distribution with the flow field produces oscillations in the average vertical flow component and the root mean squared velocities of both components.

\subsection{Oscillation periods}

In each measurement, the driving rate $\xi$ is kept approximately constant. One may therefore expect the system to react with a constant timescale. However, we find that during one experimental run, the oscillation period $\Delta t$ decreases with increasing temperature, as can be seen in Figs. 13 and 14. This trend has been observed in turbidity measurements with other binary mixtures by Auernhammer et al. (2005) and Vollmer et al. (2007) before. Secondly, the oscillation period depends on the driving rate. For faster temperature ramps, the oscillation period decreases. We have measured the oscillation periods of the droplet size distribution for two decades in $\xi$ for the top phase and almost three decades in $\xi$ for the bottom phase. The colors and symbols in Fig. 15 encode different heating rates $\xi$ : black stars, $\xi<6 \times 10^{-6} \mathrm{~s}^{-1}$; blue crosses, $\xi<1.3 \times 10^{-5}$ $\mathrm{s}^{-1}$; cobalt circles, $\xi<3 \times 10^{-5} \mathrm{~s}^{-1}$; green triangles, $\xi<6 \times 10^{-5} \mathrm{~s}^{-1}$; red squares, $\xi<3 \times 10^{-4} \mathrm{~s}^{-1}$; and magenta diamonds, $\xi>3 \times 10^{-4} \mathrm{~s}^{-1}$. A trend of the period $\Delta t$ with the driving $\xi$ is visible, but it is masked by the temperature dependence of $\Delta t$.

\section{Discussion and conclusions}

To investigate the physics of phase separation, we study the water/isobutoxyethanol system. It has very convenient 

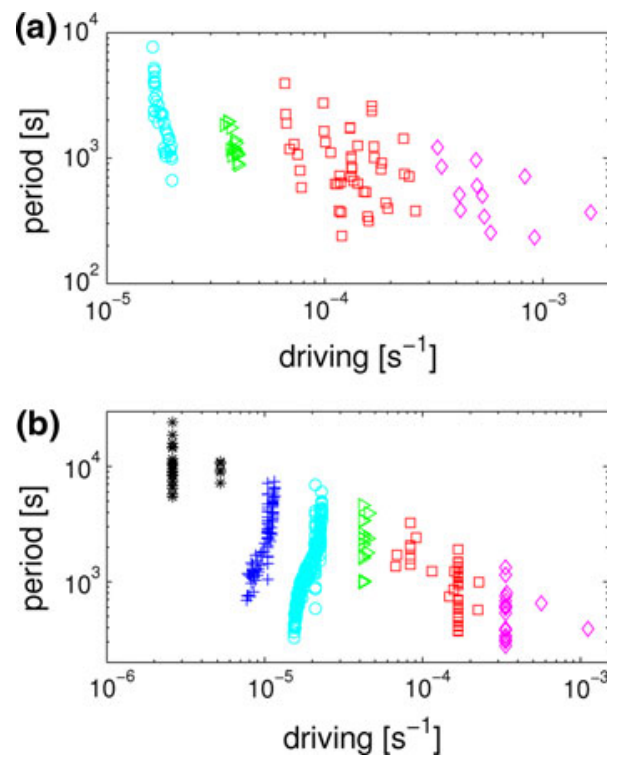

Fig. 15 Oscillation periods $\Delta t$ as a function of the driving rate $\xi$ for the top (a) and the bottom (b) phase. In both phases, the oscillation period is smaller for higher driving rates. In each experimental run, the driving is kept approximately constant. Nevertheless, the oscillation period decreases with increasing temperature. The use of the symbols is described in the text

physical properties, a critical point just above room temperature, an upper miscibility gap and low vapor pressure. Therefore, sample preparation is easy, and a broad range of parameters can be studied with a fully automatized setup. Using Nile Red as a dye and choosing a system with one polar and one organic phase, we obtained images with a high fluorescence contrast.

A short arc mercury vapor lamp is suitable for the illumination of the sample. To follow the evolution of the broad droplet size distribution for several hours, high-resolution images (5 M pixel) are taken at small frame rates $(0.3-5 \mathrm{~Hz})$. The resolution of the camera has to be large enough to detect droplet radii, whereas the frame rate must be adapted to the maximal droplet velocities. For measuring well-resolved flow fields, the droplet number density has to be high enough. This sets a lower bound to the rate of temperature change, which controls the nucleation of droplets. On the other hand, for high droplet number densities, measurements are only possible close to the walls. As the droplets are not index matched, clear images can only be obtained at a wall distance up to a few times the mean distance of droplets. Therefore, the measurements were conducted $0.3 \mathrm{~mm}$ away from the wall, that is, at a distance of about 1.5 times the correlation length calculated from the flow fields.

We presented a particle tracking algorithm based on image processing with MATLAB. It detects droplets in a range of 4 to $40 \mu \mathrm{m}$ radius (bigger ones can be detected but are very scarce) in a sample field of $1.3 \times 1.5 \mathrm{~mm}^{2}$. For the detection of droplets in single images, a threshold-based and a watershed-based image segmentation algorithms are implemented. The former one works well for big droplets and only slightly overlapping droplet images. With the latter one, even small droplets in front of big ones can be detected, but big droplets are frequently oversegmented. A match function derived from the radial intensity gradient at the edge of a droplet image is used to evaluate and optimize the droplet detection in images. The tracking of droplets is a key step in distinguishing real droplets from artefacts.

The motion of the droplets is decomposed into a sedimentation and an advection term. The sedimentation velocity is given by the Stokes velocity of a sphere. The advection of droplets is sampled on an Eulerian grid. By taking the sedimentation velocity explicitly into account, big droplets can also be used to calculate the flow field, which reduces the noise. This is possible because the Stokes number is much smaller than one for all droplets in our experiments. It is not necessary to know in advance the radius at which sedimentation starts to become significant. This threshold depends on the specific flow conditions.

There are two advantages by incorporating information on the droplet radius: First, the radius is a good criterion to identify droplets in subsequent images. Secondly, the prediction of the droplet position in the next image is improved by knowledge of the radius-dependent sedimentation velocity.

The experimental setup and the tracking algorithm are optimized for long measurement times (several hours) and low Reynolds number flow. Our experimental procedure can be applied to a broad variety of binary mixtures, provided an appropriate fluorescent dye for labeling (only) one of the two phases is found and the excitation and emission filters are adapted to it. It enables then detailed investigations of the evolution of the system: The droplet size distribution, Lagrangian particle velocities and Eulerian flow fields can be measured simultaneously for a broad range of heating rates, temperatures and sample geometries.

To investigate other laminar or turbulent flows, a highspeed camera has to be used. Our technique might then be a promising approach to investigate turbulent boundary layers with reactions or phase separation. Furthermore, one may consider placing an endoscope into the sample, as Maass et al. (2009) have done, for example, to measure the size distribution outside the boundary layer.

A further advantage of the technique is that the simultaneous measurement of particle position and particle size allows us to determine the size evolution of individual droplets as they progress across the measurement area. This information can be used to obtain information on coalescence rates and collision efficiency of sedimenting droplets: A preliminary study shows that droplets with radii 
about $40 \mu \mathrm{m}$ grow on average by $0.5 \mu \mathrm{m} / \mathrm{s}$, while they travel across the measurement area. This growth amounts to a collision efficiency (see Pruppacher and Klett 1997 p. 569; Pinsky et al. 1999) of order unity. Further studies are under way to determine how the collision efficiency depends on the radius of the sedimenting droplet. This methodology should be of interest to precipitation processes such as rain formation, where the growth of medium sized droplets is believed to be driven by coalescence.

Acknowledgments We are grateful to Wilhelm Hüttner, Konstantin Christou, Kristian Hantke, Alberto de Lozar and Eric Stellamanns for enlightening discussions and experimental tests of several illumination techniques. We thank Doris Vollmer and Günther Auernhammer for advice in designing the setup, choosing the system and developing the experimental protocol. We thank Markus Holzner and Mukund Vasudevan for comments on the manuscript. Tobias Lapp acknowledges financial support from Deutsche Forschungsgemeinschaft FOR 1182 .

Open Access This article is distributed under the terms of the Creative Commons Attribution Noncommercial License which permits any noncommercial use, distribution, and reproduction in any medium, provided the original author(s) and source are credited.

\section{Appendix}

The viscosity of i-BE is measured with an Ubbelohde viscosimeter type 537 10/I made by Schott. The temperature dependence of the viscosity $\eta[\mathrm{kg} / \mathrm{ms}]$ is fitted by

$\eta(T)=A \times 10^{\frac{B \cdot(20-T)-C \cdot(T-20)^{2}}{T+D}}$

with temperatures expressed in ${ }^{\circ} \mathrm{C}$. The coefficients are given in Table 1, where the values for water are taken from Weast (1988-1989).

To interpolate the viscosities for a mixed phase of given mass fraction $\Phi$ we use the composition-dependent viscosities at $25^{\circ} \mathrm{C}$ for a homogeneous mixture in the singlephase regime given in Menzel et al. (2003). The data are fitted with a fifth-order polynomial

$$
\begin{aligned}
\eta(\Phi, \mathrm{T}= & \left.25^{\circ} \mathrm{C}\right) \\
= & -40.66 \Phi^{5}+103.44 \Phi^{4}-100.32 \Phi^{3}+39.35 \Phi^{2} \\
& +0.17 \Phi+0.91 .
\end{aligned}
$$

Assuming that the coefficients of interpolation are not changing substantially in the temperature range of our

Table 1 Fit coefficients for the viscosity of water (Weast 19881989) and i-BE, defined by Eq. 10

\begin{tabular}{lllll}
\hline & $A(\mathrm{~kg} / \mathrm{ms})$ & $B$ & $C$ & $D$ \\
\hline Water & $1.002 \times 10^{-3}$ & 1.3272 & 0.001053 & 105 \\
i-BE & $3.36 \times 10^{-3}$ & 1.730 & 0.001 & 108 \\
\hline
\end{tabular}

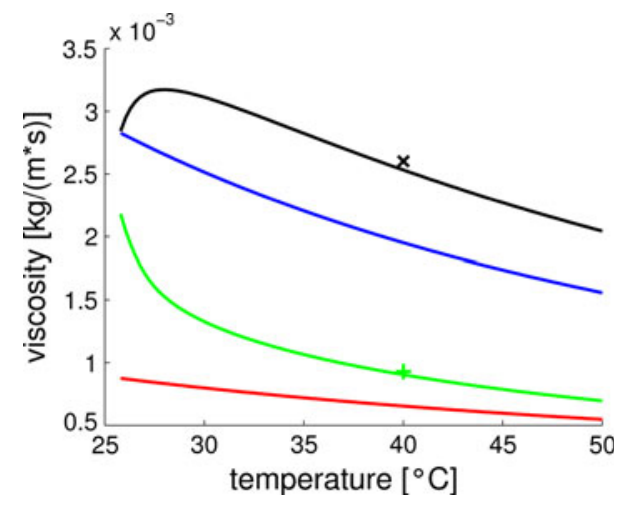

Fig. 16 From top to bottom: Viscosity of the top phase, isobutoxyethanol, the bottom phase and water, using Eqs. 10, 12. At $40^{\circ} \mathrm{C}$, the viscosity of the two mixed phases was measured with an Ubbelohde viscosimeter

measurements, a rescaled viscosity $\tilde{\eta}(\Phi)$ is defined. It only depends on the composition $\Phi$

$\eta(\Phi, T)=\tilde{\eta}(\Phi) \cdot \eta_{i B E}(T)+(1-\tilde{\eta}(\Phi)) \cdot \eta_{H_{2} O}(T)$.

The viscosities of the two phases are shown in Fig. 16 as a function of temperature. To check the strong assumption entering this interpolation, we measured the viscosity of the two phases at $T=40^{\circ} \mathrm{C}$. For both phases, it followed the prediction of Eq. 12 to within $2 \%$. This is sufficiently accurate for our means.

\section{References}

Aarts DGAL, Dullens RPA, Lekkerkerker HNW (2005) Interfacial dynamics in demixing systems with ultralow interfacial tension. New J Phys 7:40

Auernhammer GK, Vollmer D, Vollmer J (2005) Oscillatory instabilities in phase separation of binary mixtures: fixing the thermodynamic driving. J Chem Phys 123(13):134, 511-518

Aveyard R, Binks BP, Clint JH (2003) Emulsions stabilised solely by colloidal particles. Adv Colloid Interface Sci 100-102:503-546

Batchelor G (2005) An introduction to fluid dynamics. Cambridge University Press, Cambridge

Benczik IJ, Vollmer J (2010) A reactive-flow model of phase separation in fluid binary mixtures with continuously ramped temperature. Europhys Lett 91(3):36 003

Binks BP (2002) Particles as surfactants—similarities and differences. Curr Opin Colloid Interface Sci 7(1-2):21-41

Cates ME, Vollmer J, Wagner A, Vollmer D (2003) Phase separation in binary fluid mixtures with continuously ramped temperature. Philos Trans R Soc Lond Ser A 361(1805):793-807

Doi H, Tamura K, Murakami S (2000) Thermodynamic properties of aqueous solution of 2-isobutoxyethanol at $\mathrm{T}=(293.15,298.15$, and 303.15) K, below and above LCST. J Chem Thermodyn 32(6):729-741

Emmanuel S, Berkowitz B (2006) An experimental analogue for convection and phase separation in hydrothermal systems. J Geophys Res 111:B09103

Fowler S, Greenspan P (1985) Application of Nile red, a fluorescent hydrophobic probe, for the detection of neutral lipid deposits in 
tissue sections: comparison with oil red O. J Histochem Cytochem 33(8):833-836

Gonzalez RC, Woods RE, Eddins S1 (2004) Digital image processing using MATLAB. Pearson Education Inc., New Jersey

Hayase Y, Kobayashi M, Vollmer D, Pleiner H, Auernhammer GK (2008) Asymmetric oscillations during phase separation under continuous cooling: a simple model. J Chem Phys 129(18): 184,109-117

Heffels C, Polke R, Rädle M, Sachweh B, Schäfer M, Scholz N (1998) Control of particulate processes by optical measurement techniques. Part Part Syst Charact 15(5):211-218

$\mathrm{Hu}$ B, Angeli P, Matar OK, Lawrence CJ, Hewitt GF (2006) Evaluation of drop size distribution from chord length measurements. AlChE J 52(3):931-939

Kreizer M, Ratner D, Liberzon A (2010) Real-time image processing for particle tracking velocimetry. Exp Fluids 48(1):105-110

Krishnamurthy S, Goldburg WI (1980) Kinetics of nucleation in a binary liquid mixture. Phys Rev A 22:2147-2155

Lovick J, Mouza A, Paras S, Lye G, Angeli P (2005) Drop size distribution in highly concentrated liquid/liquid dispersions using a light back scattering method. J Chem Technol Biotechnol 80(5):545-552

Maaß S, Wollny S, Voigt A, Kraume M (2011) Experimental comparison of measurement techniques for drop size distributions in liquid/liquid dispersions. Exp Fluids 50(2):259-269

Maas HG, Gruen A, Papantoniou D (1993) Particle tracking velocimetry in three-dimensional flows. Exp Fluids 15(2):133-146

Maass S, Grünig J, Kraume M (2009) Measurement techniques for drop size distributions in stirred liquid-liquid systems. Chem Process Eng 30:635-651

Malik NA, Dracos T, Papantoniou DA (1993) Particle tracking velocimetry in three-dimensional flows. Exp Fluids 15(4):279-294

Menzel K, Mirzaev SZ, Kaatze U (2003) Crossover behavior in micellar solutions with lower critical demixing point: broadband ultrasonic spectrometry of the isobutoxyethanol-water system. Phys Rev E 68(1):011501

Nakayama H, Kanenaga H, Fujioka T (2001) The study of water structure in aqueous solutions of butoxyethanol by enthalpy of mixing measurements. J Therm Anal Calorim 64(1):193-199
Ouellette N, Xu H, Bodenschatz E (2006) A quantitative study of three-dimensional Lagrangian particle tracking algorithms. Exp Fluids 40(2):301-313

Petrak D (2002) Simultaneous measurement of particle size and particle velocity by the spatial filtering technique. Part Part Syst Charact 19(6):391-400

Pinsky M, Khain A, Shapiro M (1999) Collisions of small drops in a turbulent flow. part I: collision efficiency. Problem formulation and preliminary results. J Atmos Sci 56(15):2585-2600

Pruppacher HR, Klett JD (1997) Microphysics of clouds and precipitation. 2nd edn. Kluwer Academic Publications, The Netherlands

Ruf A, Worlitschek J, Mazzotti M (2000) Modeling and experimental analysis of PSD measurements through FBRM. Part Part Syst Charact 17(4):167-179

Sagui C, Grant M (1999) Theory of nucleation and growth during phase separation. Phys Rev E 59(4):4175-4187

Simmons MJH, Zaidi SH, Azzopardi BJ (2000) Comparison of laserbased drop-size measurement techniques and their application to dispersed liquid-liquid pipe flow. Opt Eng 39:505

Thijssen JHJ, Clegg PS (2010) Emulsification in binary liquids containing colloidal particles: a structure-factor analysis. J Phys Condens Mat 22(45):455102

Vaillancourt PA, Yau MK (2000) Review of particle-turbulence interactions and consequences for cloud physics. Bull Am Meteorol Soc 81(2):285-298

Vollmer D, Vollmer J, Strey R (1997) Oscillations in the dynamics of temperature-driven phase separation. Europhys Lett 39(3):245250

Vollmer D, Vollmer J, Wagner AJ (2002) Oscillatory kinetics of phase separation in a binary mixture under constant heating. Phys Chem Chem Phys 4:1380-1385

Vollmer J (2008) Phase separation under ultraslow cooling: onset of nucleation. J Chem Phys 129(16):164502

Vollmer J, Auernhammer GK, Vollmer D (2007) Minimal model for phase separation under slow cooling. Phys Rev Lett 98(11): 115701

Weast RC (1988-1989) CRC Handbook of chemistry and physics, 69th edn. CRC Press, Boca Raton 